\title{
METODOLOGIA PARA AVALIAÇÃO DE ESPECTROFOTÔMETROS: ANÁLISE DA DISPERSÃO DAS MEDIDAS
}

\author{
Cleonilde Maria do Nascimento ${ }^{1 *}$, Carla Luiza Barros Bernardes Borja ${ }^{1}$, Bruno Edberg Alves de \\ Lira $^{1}$, Jabson Herber Profiro de Oliveira ${ }^{2}$, Dijanah Cota Machado ${ }^{3}$, Milton Marcelino Filho ${ }^{3}$ \\ ${ }^{1}$ Monitor da disciplina de Física e Biofísica-UFPE, ${ }^{2}$ Mestrado em Inovação Terapêutica-UFPE, \\ ${ }^{3}$ Departamento de Biofísica e Radiobiologia-UFPE \\ *cl.manasci@gmail.com
}

\section{INTRODUÇÃO}

A investigação científica na área das ciências da vida, associada aos conhecimentos da química e da física, permitiu o desenvolvimento de inúmeras técnicas e metodologias analíticas (CISTERNAS; VARGAS; MONTE, 2005). A espectrofotometria está entre estas técnicas e é amplamente utilizada em laboratórios de análises clínicas e laboratórios de pesquisa, principalmente nas investigações bioquímicas, físicas, químicas e farmacológicas (CERRI et al., 2017). A espectrofotometria, juntamente com a fotocolorimetria, é um método biofísico que se baseia na relação existente entre a absorção de luz e a concentração de uma substâ ncia em solução, permitindo determinar as concentrações de espé cimes químicas diversas, como carboidratos, proteínas, lipídios, colesterol entre outras (HIRANO et al., 2001). No âmbito clínico, a análise quantitativa de biomoléculas presentes nos fluidos bioló gicos (urina, plasma ou soro) é essencial para a compreensão de disfunções metabólicas desencadeadas por alguma patologia.

Por meio da espectrofotometria, aplicada às análises clínicas e por outras tecnologias, diretrizes da área médica foram sendo estabelecidas para a condução de um diagnóstico mais consistente e assertivo, possibilitando assim um encaminhamento terapêutico eficaz (CERRI et al., 2017).

Além das inúmeras aplicações nas análises clínicas e na pesquisa científica, a espectrofotometria pode ser também aplicada na área odontológica. 0 espectrofotômetro está entre os instrumentos mais precisos, úteis e de fácil utilização para mensuração de cor, sendo capaz de fornecer o espectro de emissão de luz dos dentes vitais ou extraídos e dos materiais restauradores (DIESEL, 2011).

A espectrofotometria também é utilizada em diversos setores da indústria, principalmente têxtil, alimentício, químico e farmacê utico, que utilizam em seus produtos e processos os mais variados tipos de corantes. Alguns corantes sintéticos utilizados em alimentos, como também em medicamentos e cosméticos, apresentam potencial toxicológico e por conta disto se faz necessá ria a realização de medidas espectrofotométricas para o controle de qualidade de tais compostos (SANTOS et al., 2010). No Brasil, a utilização destes corantes é normatizada pela ANVISA (Agência de Vigilância Sanitária) (BRASIL, 1977).

Diante da ampla aplicabilidade do espectrofotômetro, é importante se ter uma boa capacitação teórico-prática dos usuá rios, bem como métodos para avaliar os indicadores de qualidade do equipamento, garantindo assim, a obtenção de resultados experimentais ou clínicos satisfatórios.
O objetivo do presente estudo foi fomentar a experiência de alunos com a prática de biofísica, com as técnicas de estatística e com os métodos de obtenção de indicadores de qualidade, por meio da realização de experimentos laboratoriais utilizando espectro-fotô metros, mais especificamente, analisando a dispersão das medidas de absorbância apresentadas por estes equipamentos.

\section{MATERIAIS E MÉTODOS}

Foram avaliados quatro espectrofotômetros da marca Kasuaki ${ }^{\circledast}$, modelo IL-226 (Figura 1), rotineiramente empregados nas aulas prá ticas da disciplina Física e Biofísica 1, ministrada para os cursos das áreas de saúde e biológicas da Universidade Federal de Pernambuco-UFPE. Os números de série de cada equipamento são: 20152166 (equipamento 1); 20152167 (equipamento 2); 20152168 (equipamento 3); e 20152169 (equipamento 4).

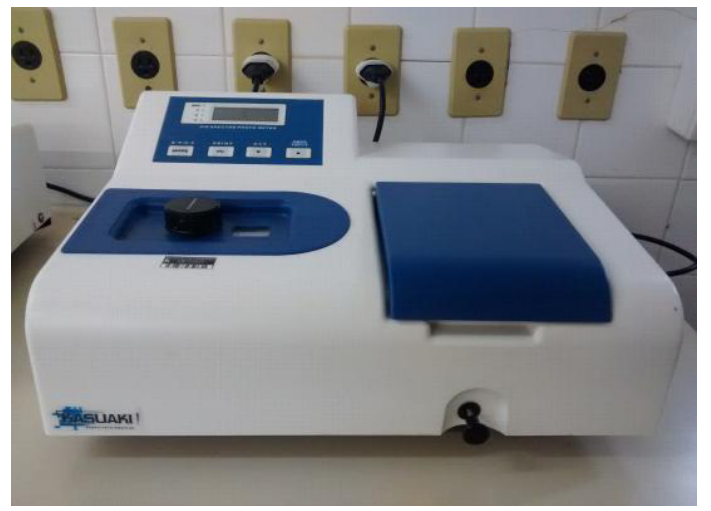

Figura 1: Vista externa do espectrofotômetro, marca Kasuaki ${ }^{\oplus}$, modelo IL-226.

Para as medidas de absorbância, foram utilizadas cubetas de vidro e uma solução aquosa do corante vermelho neutro a 0,00125\%. Para calibrar o "zero" de absorbância utilizou-se como "branco" uma cubeta contendo água destilada. Todos os equipamentos foram ligados dez minutos antes de iniciar os testes, tempo requerido para estabilizá-los. Após este tempo, foi selecionado o comprimento de onda de $520 \mathrm{~nm}$ nos espectrofotômetros, o qual corresponde ao fotopico da substância utilizada, motivo para sua escolha.

$\mathrm{Na}$ tentativa de diminuir o erro das medidas experimentais, foi utilizada uma única cubeta para todas as medidas. Em cada 
medida, adicionava-se $3 \mathrm{~mL}$ de vermelho neutro a 0,00125\% à cubeta e em seguida a colocava no primeiro compartimento do porta-cubeta do primeiro equipamento. Após a leitura, retirava-se a cubeta e a colocava no segundo equipamento, este procedimento se repetia até chegar-se ao quarto equipamento.

Como foi observado que a localização lateral da cubeta no portacubeta altera de modo significativo os valores medidos para a absorbância, padronizou-se a posição de inserção da cubeta neste suporte, sendo todas as medidas realizadas com a cubeta posicionada na porção mais à esquerda do porta-cubeta (Figura 2). Ao final desta rodada de medidas, desprezava-se a amostra e adicionava-se uma nova amostra à cubeta, repetindo-se o procedimento descrito acima. Para realização de uma adequada análise estatística, foram feitas trinta e cinco medidas de absorbâ ncia em cada um dos equipamentos. Na leitura dos valores de absorbância foram consideradas três casas decimais. As medidas foram obtidas por quatro estudantes que receberam simultaneamente o mesmo treinamento de um instrutor técnico.

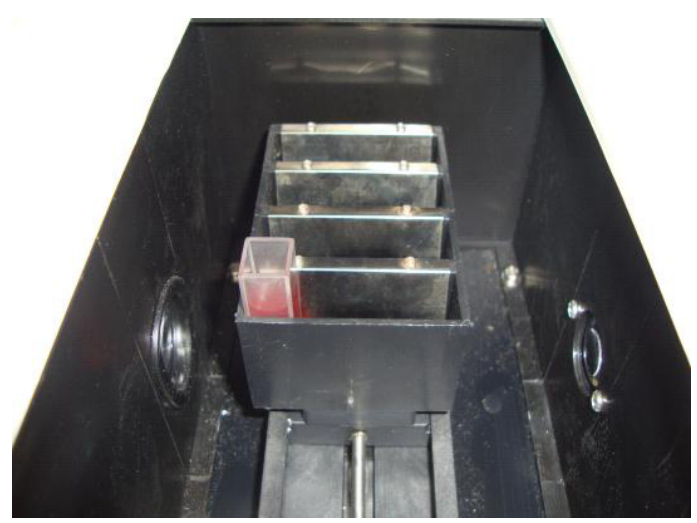

Figura 2: Posicionamento da cubeta no porta-cubeta.

Os dados obtidos foram inicialmente analisados, quanto à normalidade de sua distribuição, utilizando-se o teste de ShapiroWilk (nível de significância de $0,05 \%$ ) e estatística descritiva incluindo mediana, intervalo interquartil (IIQ) e intervalo de confiança da média para as amostras com distribuição normal. $\mathrm{Na}$ análise estatística foi utilizado o programa R CRAN v.3.4.2.

\section{RESULTADOS E DISCUSSÃO}

Para avaliar o espalhamento dos dados obtidos foi utilizada a medida de dispersão da absorbância. Destaca-se que dispersão dos dados é um conceito que diz respeito a como e quanto os dados estão próximos ou distantes entre si.

Nos resultados obtidos foram encontrados quatro outliers (medidas atípicas), dois nas medidas realizadas com o equipamento 2 e dois nas medidas com o equipamento 3. Estes foram excluídos do conjunto de dados. Em sequência foram obtidos os seguintes resultados:

As medidas obtidas no equipamento 1 tiveram uma mediana de 0,6910 . Como evidenciado na figura 3 , os dados não apresentaram distribuição normal. Fato confirmado através do teste estatístico de Shapiro-Wilk ( $\mathrm{p}$-valor $<0,05)$.

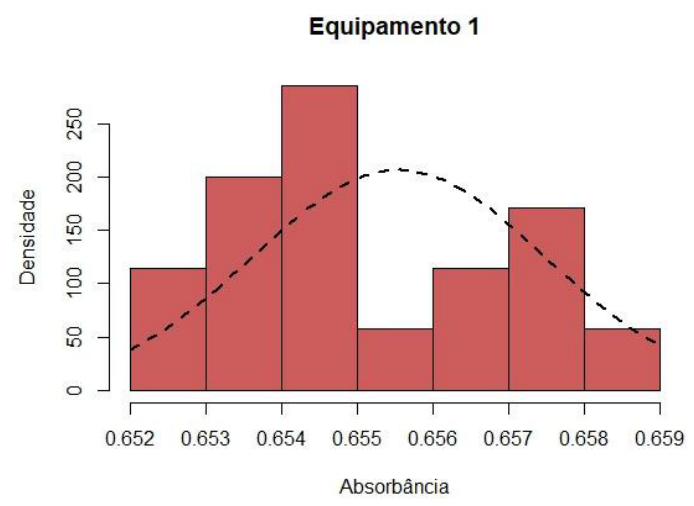

Figura 3: Distribuição de frequência das medidas obtidas com o equipamento 1 ( $n=35)$.

As medidas obtidas no equipamento 2 tiveram uma mediana de 0,6560 . Como evidenciado na figura 4 e comprovado estatisticamente, os dados não apresentaram distribuição normal, (p-valor $>0,05)$.

Equipamento 2

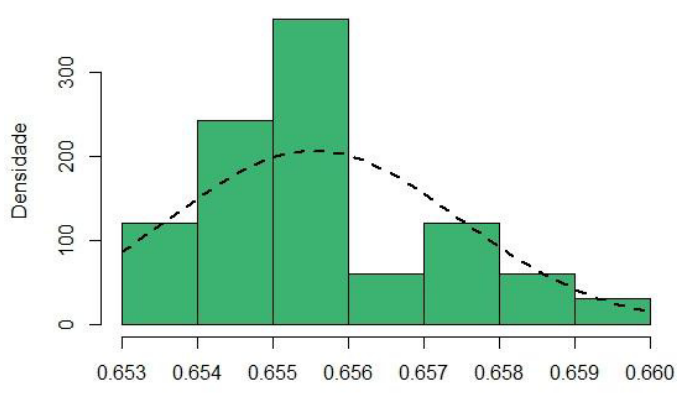

Absorbância

Figura 4: Distribuição de frequência das medidas obtidas com o equipamento 2 ( $n=33)$.

As medidas obtidas no equipamento 3 tiveram uma mediana de 0,6280 . Como evidenciado na figura 5 e comprovado estatisticamente, os dados apresentaram distribuição normal, ( $p$-valor $>0,05)$. Com $95 \%$ de confiança pode-se dizer que a média da população de medidas do equipamento $3(0,627727)$ encontra-se entre 0,62725 e 0,62819 .

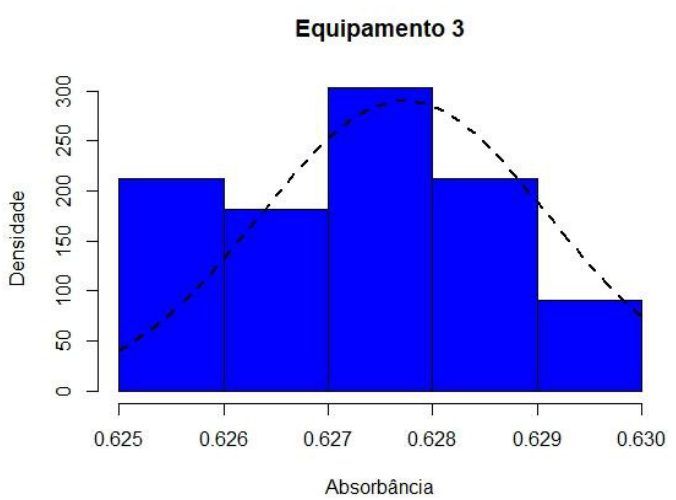

Figura 5: Distribuição de frequência das medidas obtidas com o equipamento $3(n=33)$. 
As medidas obtidas no equipamento 4 tiveram uma mediana de 0,6510. Como evidenciado na figura 6 e comprovado estatisticamente, os dados apresentaram distribuição normal, ( $p$-valor $>0,05$ ). Com $95 \%$ de confiança pode-se dizer que a média da população de medidas do equipamento $4(0,650428)$ encontra-se entre 0,64969 e 0,65115 .

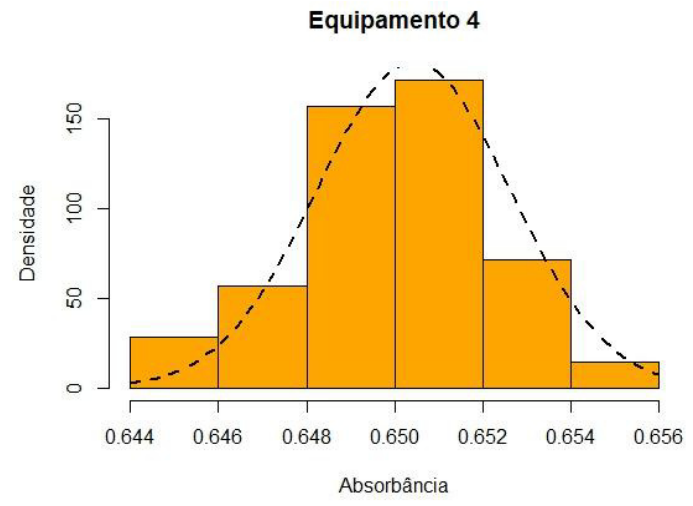

Figura 6: Distribuição de frequência das medidas obtidas com o equipamento 4 (n=35).

O quadro 1 mostra um resumo da análise estatística dos dados. Pode-se observar que o intervalo interquartil (IIQ) apresentou uma pequena amplitude, isto aconteceu como consequência da pouca dispersão das medidas.

Quadro 1: Análise estatística obtida para cada um dos quatro equipamentos.

\begin{tabular}{|c|c|c|c|c|}
\hline Equipamentos & $\mathbf{n}$ & Mediana & $\begin{array}{c}\text { Distribuição } \\
\text { normal ? }\end{array}$ & IIQ \\
\hline \hline EQ1 & 35 & 0,6550 & Não & 0,003 \\
\hline EQ2 & 33 & 0,6560 & Não & 0,002 \\
\hline EQ3 & 33 & 0,6280 & Sim & 0,002 \\
\hline EQ4 & 35 & 0,6510 & Sim & 0,003 \\
\hline
\end{tabular}

$\mathrm{Na}$ figura 7 pode-se visualizar de outra forma o comportamento da dispersão dos dados e suas respectivas medianas, representadas pela linhas mais escuras nas caixas do gráfico. Com destaque para a semelhança das medianas do equipamento 1 e do equipamento 2 e para uma discrepância da mediana do equipamento 3 , em relaçã o aos demais. Ressalta-se também o pequeno grau de dispersão das medidas realizadas pelos quatro equipamentos, como pode ser observado pelos intervalos interquartis.

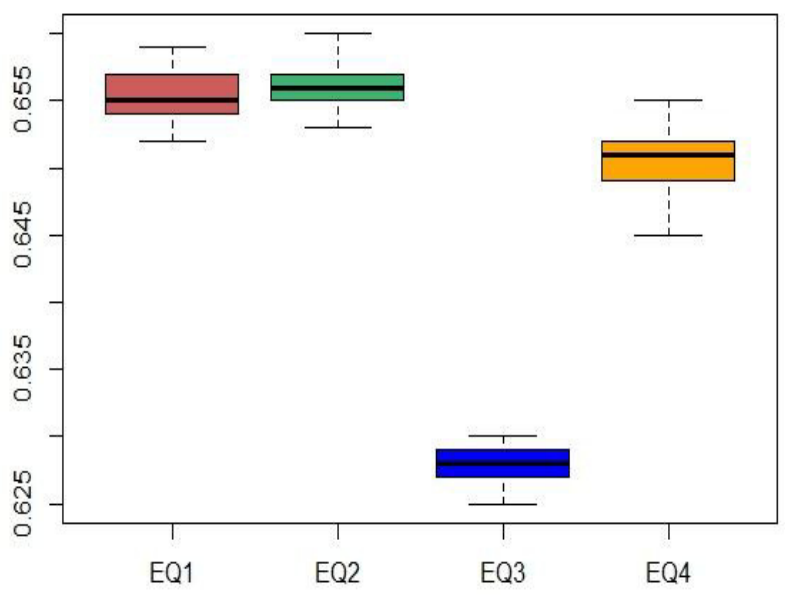

Figura 7: Boxplots comparativos dos dados obtidos com os equipamentos.
A literatura em estatística aponta o uso de boxplot como uma das alternativas para identificar-se valores discrepantes (outliers) (BARNETT; LEWIS, 1994; SCHWERTMANA; OWENSA; ADNANB, 2004; CARTER; SCHWERTMAN; KISER, 2009; LI et al., 2016), que muito provavelmente são frutos de erros esporádicos nas medições. $\mathrm{Na}$ análise do boxplots, as caixas com amplo "corpo" demonstram valores espalhados para as medidas. Estes, quando não controlados, podem vir a influenciar no resultado final de testes estatísticos e consequentemente na conclusão da pesquisa.

Uma técnica que pode ser aplicada com o objetivo de complementar a avaliação dos dados é a análise de variância (ANOVA), ou ainda, seu equivalente para quando os dados não possuírem distribuição normal, o teste Kruskall Wallys. Em caso de constatação de diferença estatística em pelo menos um grupo (amostra), deve-se aplicar um teste para identificação do grupo ou dos grupos que diferem dos demais.

No presente estudo estes testes não foram aplicados pelo fato de existir um considerável número de medidas de mesmo valor (empates), consequentemente, não possibilitando estas análises complementares.

Contudo, todas estas ferramentas são recursos úteis para o conhecimento do funcionamento de equipamentos utilizados em pesquisa acadêmica. 0 contato de alunos de graduação com estes conceitos favorece a familiaridade com estas metodologias.

Entretanto, há necessidade de uma ampliação na análise das medidas realizadas por estes equipamentos, avaliando-se outras características, igualmente importantes, dos equipamentos como a exatidão, a sensibilidade e a reprodutibilidade. Os dados obtidos sugerem existir uma diferença na exatidão dos equipamentos. Assim sugerimos, como desdobramento deste estudo, a análise dos conceitos de exatidão, sensibilidade e reprodutibilidade. Cabe ressaltar que este não consistiu em um objetivo do presente trabalho.

\section{CONCLUSÕES}

0 presente trabalho representa uma iniciativa positiva no sentido de promover a capacitação dos alunos de graduação da disciplina de Biofísica da UFPE, proporcionando uma avaliação crítica na obtenção de medidas com equipamentos de laboratório.

Os alunos constataram na prática que os equipamentos apresentaram medidas com pequena dispersão. Entretanto, observou-se a necessidade de investigação da exatidão destes equipamentos.

\section{REFERÊNCIAS}

ALVES, J.K.G.; AUED, N.; SOARES, F.Z.M.; JACQUES, L.B.; KAIZER, M.R.; MALLMANN, A. Avaliação de cor em compósitos com o espectrofotômetro Easyshade. RFO, Passo Fundo, v. 19, n. 1, p. 101-106, 2014.

BARNETT V.; LEWIS,T. Outliers in statistical data. John Wiley, 1994.

BRASIL, Comissão Nacional de Normas e Padrões para Alimentos, Ministério da Saúde. Resolução - CNNPA nº 44, 1977.

CARTER, N.J.; SCHWERTMAN, N.C.; KISER, T.L. A comparison of two boxplot methods for detecting univariate outliers which adjust for sample size and asymmetry. Statistical Methodology, v. 6, p. 604-621, 2009.

CERRI, G.G.; JATENE, F.B.; NOBRE, M.R.; CUCE, BERNARDO, W.M. Introdução. Projeto Diretrizes. Associação Médica Brasileira e Conselho Federal de Medicina. Disponível em <http://www.projetodiretrizes.org.br/projeto_diretrizes/texto_int rodutorio.pdf> Acesso em 19 de maio de 2017.

CISTERNAS, J.R.; VARGAS, J.; MONTE, O. Fundamentos de Bioquí mica Experimental. São Paulo: Editora Atheneu, 2005. 
DIESEL, P.G. Avaliação da cor de resinas compostas em meios e tempos de armazenamentos distintos usando dois espectrofotô metros. 07 de fevereiro de 2011, 61 folhas. Dissertação de Mestrado. Universidade Federal de Santa Maria. RS, Brasil, 2011.

HIRANO, Z.M.B.; SILVA FILHO, H.H.; MULLER, G.C.K.; SCHMIDT, S.R. Bioquímica - manual prático. Blumenau: EDIFURB, 2001.

LI, A.; FENG, M.; LI, Y.; LIU, Z. Application of Outlier Mining in Insider Identification Based on Boxplot Method. Procedia Computer Science, v. 91, p. 245-251, 2016.

MOTTA, V.T. Bioquímica Clínica para Laboratório - princípios e interpretações. $5^{\text {a }}$ edição. Rio de Janeiro: MedBook, 2009.

SANTOS, M.E.D.; DEMIATE, I.M.; NAGATA, N. Determinação simultâ nea de amarelo tartrazina e amarelo crepúsculo em alimentos via espectrofotometria UV-VIS e métodos de calibração multivariada. Ciência e Tecnologia de Alimentos, v. 30, p. 903-909, 2010.

SCHWERTMANA, N.C.; OWENSA, M.A.; ADNANB, R. A simple more general boxplot method for identifying outliers. Computational Statistics \& Data Analysis, v. 47, p. 165-174, 2004.

\begin{tabular}{|c|c|c|c|c|}
\hline & EQ1 & EQ2 & EQ3 & EQ4 \\
\hline 1 & 0,654 & 0,653 & 0,626 & 0,645 \\
\hline 2 & 0,657 & 0,656 & 0,627 & 0,648 \\
\hline 3 & 0,658 & 0,657 & 0,629 & 0,648 \\
\hline 4 & 0,659 & 0,658 & 0,629 & 0,649 \\
\hline 5 & 0,658 & 0,658 & 0,628 & 0,649 \\
\hline 6 & 0,657 & 0,657 & 0,629 & 0,651 \\
\hline 7 & 0,654 & 0,655 & 0,626 & 0,649 \\
\hline 8 & 0,655 & 0,655 & 0,627 & 0,648 \\
\hline 9 & 0,654 & 0,654 & 0,627 & 0,649 \\
\hline 10 & 0,652 & 0,653 & 0,625 & 0,646 \\
\hline 11 & 0,656 & 0,656 & 0,629 & 0,65 \\
\hline 12 & 0,654 & 0,655 & 0,627 & 0,649 \\
\hline 13 & 0,654 & 0,655 & 0,627 & 0,65 \\
\hline 14 & 0,654 & 0,655 & 0,626 & 0,65 \\
\hline 15 & 0,655 & 0,656 & 0,626 & 0,65 \\
\hline 16 & 0,655 & 0,655 & 0,628 & 0,651 \\
\hline 17 & 0,654 & 0,655 & 0,628 & 0,651 \\
\hline 18 & 0,655 & 0,656 & 0,628 & 0,652 \\
\hline 19 & 0,655 & 0,656 & 0,627 & 0,652 \\
\hline 20 & 0,655 & 0,656 & 0,629 & 0,651 \\
\hline 21 & 0,655 & 0,656 & 0,628 & 0,65 \\
\hline 22 & 0,655 & 0,656 & 0,628 & 0,651 \\
\hline 23 & 0,655 & 0,656 & 0,629 & 0,651 \\
\hline 24 & 0,655 & 0,656 & 0,629 & 0,651 \\
\hline 25 & 0,656 & 0,656 & 0,63 & 0,652 \\
\hline 26 & 0,658 & 0,659 & 0,628 & 0,655 \\
\hline 27 & 0,658 & 0,659 & 0,626 & 0,654 \\
\hline 28 & 0,653 & 0,656 & 0,625 & 0,651 \\
\hline 29 & 0,652 & 0,654 & 0,63 & 0,649 \\
\hline 30 & 0,653 & 0,655 & 0,628 & 0,648 \\
\hline 31 & 0,658 & 0,66 & 0,628 & 0,653 \\
\hline 32 & 0,657 & 0,658 & 0,628 & 0,652 \\
\hline 33 & 0,657 & 0,658 & 0,63 & 0,653 \\
\hline 34 & 0,658 & - & - & 0,653 \\
\hline 35 & 0,659 & - & - & 0,654 \\
\hline
\end{tabular}

\section{APÊNDICE}

